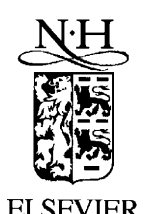

Physica A 299 (2001) 1-15

\title{
PHYSICA
}

ELSEVIER

Physica A $299(2001)$ 1-15

www.elsevier.com/locate/physa

\section{Similarities and differences between physics and economics}

\author{
H.E. Stanley ${ }^{\mathrm{a}, *}$, L.A.N. Amaral ${ }^{\mathrm{a}}$, X. Gabaix ${ }^{\mathrm{b}}$, \\ P. Gopikrishnan ${ }^{\mathrm{a}}, \mathrm{V}$. Plerou ${ }^{\mathrm{a}}$ \\ ${ }^{a}$ Center for Polymer Studies and Department of Physics, Boston University, Boston, MA 02215, USA \\ ${ }^{\mathrm{b}}$ Department of Economics, Massachusetts Institute of Technology, Cambridge, MA 02142, USA
}

\begin{abstract}
In this opening talk, we discuss some of the similarities between work being done by economists, and by physicists seeking to contribute to economics. We also mention some of the differences in the approaches taken, and justify these different approaches by developing the argument that by approaching the same problem from different points of view new results might emerge. In particular, we review some recent results, for example the finding that there are two new universal scaling models in economics: (i) the fluctuation of price changes of any stock market is characterized by a PDF which is a simple power law with exponent 4 that extends over $10^{2}$ standard deviations (a factor of $10^{8}$ on the $y$-axis); (ii) for a wide range of economic organizations, the histogram that shows how size of organization is inversely correlated to fluctuations in size with an exponent $\approx 1 / 6$. Neither of these two new laws has a firm theoretical foundation. We also discuss results that are reminiscent of phase transitions in spin systems, where the divergent behavior of the response function at the critical point (zero magnetic field) leads to large fluctuations. (c) 2001 Published by Elsevier Science B.V.
\end{abstract}

\section{Introduction}

According to the economist Neil A. Chriss-who is affiliated with ICor Brokerage Incorporated in New York:

The aim of modern financial theory (or at least that part of modern finance having to do with financial markets) might be described as an attempt to produce theoretical models describing the behavior of financial markets, with an eye toward causal mechanisms, statistical laws, and even predictive power. Starting with assumptions

* Corresponding author.

E-mail address: hes@bu.edu (H.E. Stanley). 
about the behavior of rational economic agent, one makes restrictions on the set of possible laws describing financial market. Adding simplifying assumptions such as frictionless markets, an absence of transaction costs, and unlimited short selling, the analysis is brought into the realm of the tractable. By observing the behavior of actual financial markets, through the collection and analysis of time series of financial data, one ultimately eliminates many models that are a priori possible but contrary to observed behavior [1].

Thus one prevalent paradigm in economics is to mary finance with mathematics, with the fruit of this marriage the development of models. In physics, we also develop and make use of models (or "artificial worlds"). However a large number of physicists are fundamentally empirical in our approach to science-indeed, some physicists never make reference to models at all (other than in classroom teaching situations). This empirical approach has led to advances when theory has grown out of experiment; one such example is the understanding of phase transitions and critical phenomena. Such a basic and deep grounding in empirical facts could have an influence on the way physicists approach economics. Our approach has been to follow the paradigm of critical phenomena, which also studies complex systems comprised of many interacting subunits, i.e., to first examine the empirical facts as thoroughly as possible before we begin to construct models.

\section{Fluctuations in finance}

A physicist views the economy as a collection of interacting units. This collection is complex; everything depends on everything else. The interesting problem is: how does everything depend on everything else? Physicists are looking for empirical laws that will describe, and theories that will help understand, this complex interaction [2-5].

To a physicist, the most interesting thing about economics is that it is dominated by fluctuations, e.g., stock averages such as the S\&P 500. If we make a curve of the values of this index over a 35-year period, we see a fluctuating signal (Fig. 1a). Statistical physicists are particularly interested in fluctuating signals. The nature of this fluctuation immediately suggests to a physicist a model that was developed 100 years ago by Bachelier: the biased random walk [6].

A one-dimensional random walk is a drunk with a coin and a metronome. At each beat of the metronome the coin is flipped-heads means one step to the right, tails one step to the left. If we look at our S\&P 500 plot placed alongside a graph of a one-dimensional biased random walk-it is biased because it uses a "biased coin" that has a slight tendency to go up rather than down-we physicists see a reasonable visual similarity (Fig. 1b). In fact, many economic pricing models-e.g., Black and Scholes - use this biased random walk in a slightly dressed-up form.

But there are certain points in the S\&P 500 plot, such as October 19, 1987, that are not mirrored anywhere in the biased random walk model-nowhere do we see a 

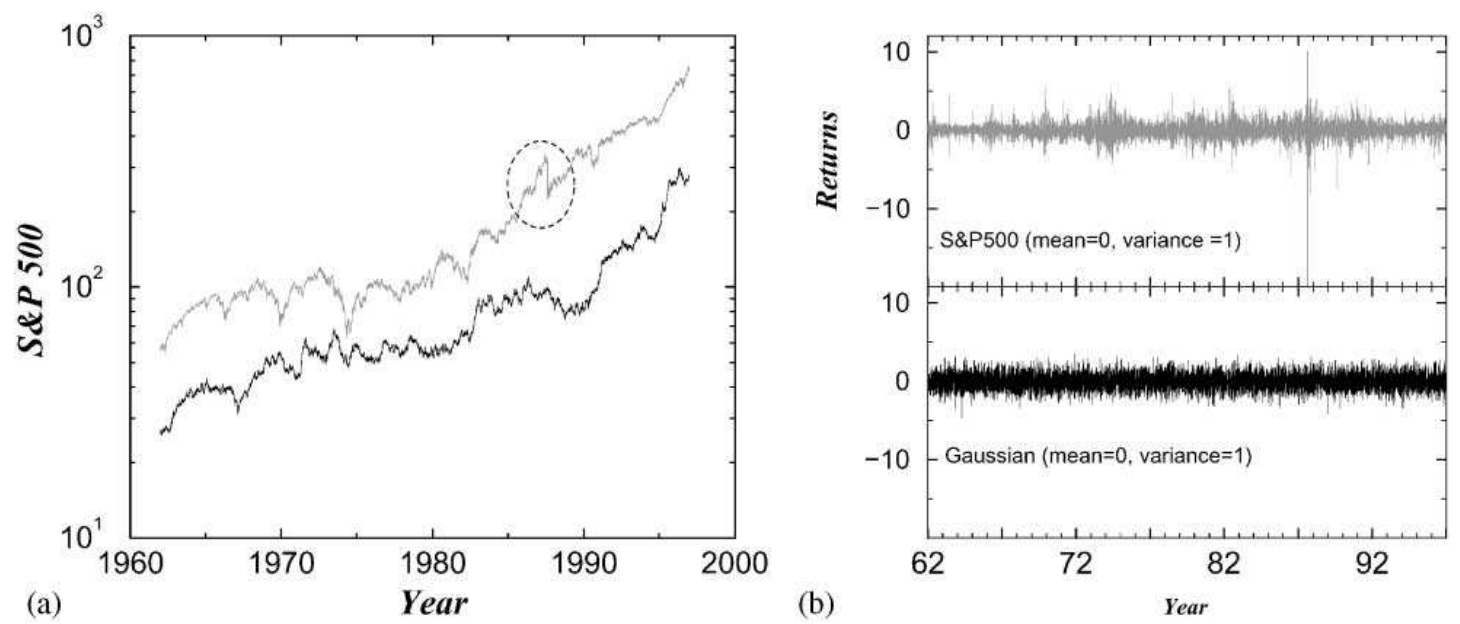

Fig. 1. (a) The S\&P 500 index is the sum of the market capitalizations of 500 companies. The sharp jump seen in 1987 is the market crash of October 19. Comparison of the time evolution of the S\&P 500 for the 35-year period 1962-96 (top line) and a biased Gaussian random walk (bottom line). The random walk has the same bias as the S\&P 500-approximately 7\% per year for the period considered. (b) Sequence of 10-min returns for the S\&P 500, normalized to unit variance, compared with sequence of i.i.d. Gaussian random variables with unit variance, which was proposed by Bachelier as a model for stock returns. Note that, in contrast to the top curve, there are no "extreme" events in the bottom curve.

drop anywhere near the 30 percent drop of Black Monday. This could not occur in a biased random walk - the probability that a walk will move two steps in the same direction is $p^{2}$, three steps is $p^{3}$, and so on-so the probability of many steps in the same direction is exponentially rare, virtually impossible.

Then how do we quantify these S\&P 500 fluctuations? We begin by graphing the values of the fluctuations as a function of time. We place the plot of the empirical data next to the predictions of Bachelier's model (Fig. 1b). The fluctuations in the model are normalized by one standard deviation. Note that the biased random walk has a PDF that is a Gaussian, so the probability of having more than five standard deviations is essentially zero-you can see that a line drawn at five standard deviations is outside the range of the fluctuations.

If we normalize the empirical data we see a difference. A line drawn at five standard deviations is not outside the range of the fluctuations - there are many "shocks" that exceed five standard deviations. A bar placed on the positive side at five standard deviations also has 30 or 40 hits-fluctuations that equal or exceed five standard deviations in the positive direction. Some, such as Black Monday, are more than 34 standard deviations. The exponential of $(-1 / 2)(34)^{2}$ is approximately $10^{-267 / 2}$.

Because big economic shocks affect the economy around the world ("everything depends on everything else"), the possibility of an economic "holocaust" or "meltdown" is one that we must take seriously. Big changes in stocks affect not only people with large amounts, but also those who have very little - those on the margins of society. One person's portfolio collapse is another's physical starvation; e.g., literal starvation in some areas was one result of the recent Indonesian currency collapse. 
Another example is the recent Merriwether long term capital management (LTCM) collapse, caused in part by the use of models that do not take into account those catastrophic rare events. Thus there are many reasons we physicists might be interested in understanding economic fluctuations.

\section{One possible conceptual framework}

We shall see that our analysis of empirical data shows that those catastrophic rare events are a part of the overall picture - that they are not simply inexplicable disasters beyond any possible understanding. Although this sounds as though we physicists think we can contribute to economics, it is possible that the converse may turn out to be even more true. If we join economists in studying economics, we may stumble onto some ideas that will help us back in our more traditional research areas of physics. An example is turbulence. If I stir a bucket of water, energy is added to the system on a big scale. This energy then dissipates over progressively smaller scales. This is an unsolved physics problem; many empirical facts can be stated, but little can be said about understanding it [7-9].

One could hypothesize that the economy is perhaps analogous to this example of turbulence. One can add information on a big scale to an economic system-e.g., the news of who wins a presidential election - and that information is dissipated on smaller and smaller scales. The way that you handle the "turbulence" associated with this dissipation of information in a financial market may help us understand how to approach turbulence in our physics research. As attractive as this analogy might appear intuitively, it is not so accurate quantitatively since the actual laws of turbulence are not at all the same as the empirical laws of economics [8,9], despite early claims to the contrary [7].

Almost all physics comes down to solving some kind of differential equation, i.e., most functions in physics have some kind of characteristic scale. Once you have determined the scale, you can express the function in some kind of exponential form-which has the wonderful property that the derivative of the function is also an exponential. In particular, the parameter $r$ sets the scale of the problem. If $r$ is positive, the function grows - and tells you the doubling time for the quantity of interest. Solutions to this look like Gaussians, and Gaussians are wonderful, tractable functions.

Such systems with scales describe almost everything in nature, including disordered things. Even raindrops on a sidewalk almost always have a characteristic scale. (If I "zoom in" or "zoom out", I can find the scale.) But there is a set of systems in nature that lack a scale. This set is described by power laws.

The framework for our approach to systems with many interacting subunits is something that is usually called "scale invariance". These systems vary greatly from those systems that do have scales $[10,11]$.

We are all familiar with algebraic equations, such as $x^{2}=4$, and we know the solution is a number, \pm 2 . Most of us are also familiar with functional equations, which 
are statements, not about relations between numbers, but about the functional form. Algebraic equations have solutions that are numbers, but functional equations have solutions that are functional forms. Power-law functions are the solutions of certain functional equations of the form $f(\lambda x)=\lambda^{p} f(x)$.

In a functional equation, the converse also holds, i.e., every function that is of this power-law form also obeys this functional equation. This applies in a large number of contexts, in particular, in physical systems that have been tuned to be near critical points. An example is a binary mixture of two fluids in which the temperature has been tuned to be a special value called the critical temperature. At that temperature there occur fluctuations in density in the binary mixture that extend over all length scales up to and including the wavelength of light. If you shine a flashlight on a tube of the binary mixture, you see movement-because the density fluctuations are so big in spatial extent they become comparable to the wavelength of the light that is interacting with them. When that occurs, you see something that is visible- "critical opalescence". The same conceptual framework that describes this system appears to be able to describe economic systems [12].

\section{Quantifying finance fluctuations}

One topic we physicists are interested in is symmetry. An example of traditional symmetry is sodium chloride. I can displace the lattice two lattice constants and the configuration will remain the same. I can rotate it $90^{\circ}$, or invert it, and the configuration will remain the same. Not only are these properties fascinating to mathematicians but they are also very relevant to solid state physics. This simple symmetry and the mathematics and physics that are built on it have led to extremely useful inventions, e.g., the transistor.

The scale-invariance symmetry involved here is just as much a symmetry as the translational invariance symmetry in sodium chloride. How useful this scale-invariance symmetry will ultimately prove to be we do not know. Over the past 30 years physicists have used the theme of scale-invariance symmetry to understand systems near their critical points. Previous to this period of time, this class of problems was one no one could solve- there were many, many length scales, not just one scale. The length scales could run from one nearest-neighbor spacing out to approximately 5000 (approximately the wavelength of light). The elements that make up this system are molecules that interact only over a short range - almost entirely with nearest neighbors. But this nearest-neighbor interaction propagates a small amount of torque through the system of nearest-neighbor interactions, so that the entire system is affected somewhat.

This is beginning to sound like economics, in which "everything affects everything else". And in economics, the first thing a physicist would do is look for the correlations. If we look at a graph of the autocorrelation function, we see a measure of the quantity $G$, which is a price change over some time horizon $\Delta t$. If we look at how 

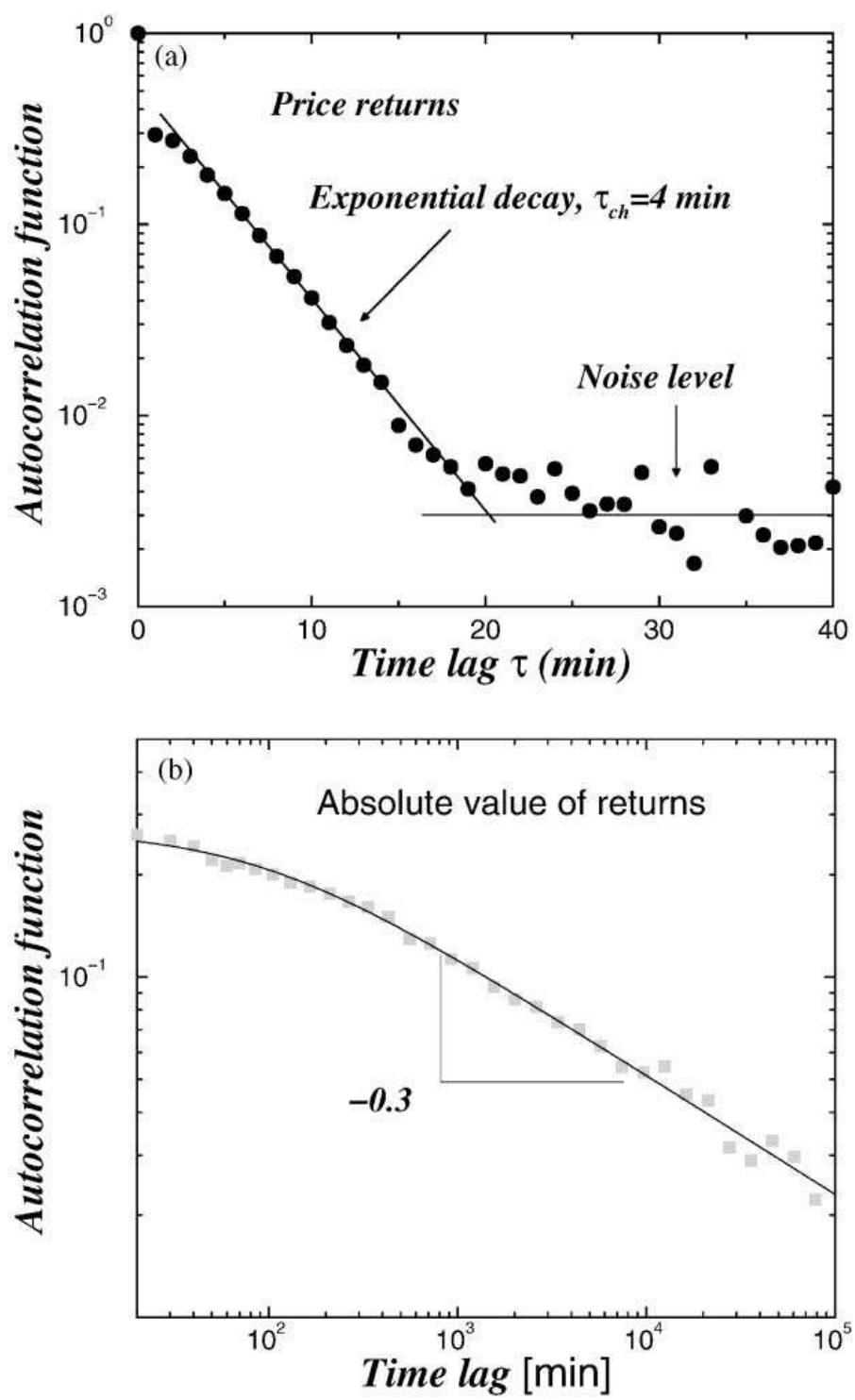

Fig. 2. (a) Semilog plot of the autocorrelation function for the S\&P 500 returns $G_{\Delta t}(t)$ sampled at a $\Delta t=1 \mathrm{~min}$ time scale, $C_{\Delta t}(\tau) \equiv\left[\left\langle G_{\Delta t}(t) G_{\Delta t}(t+\tau)\right\rangle-\left\langle G_{\Delta t}(t)\right\rangle^{2}\right] /\left[\left\langle G_{\Delta t}(t)^{2}\right\rangle-\left\langle G_{\Delta t}(t)\right\rangle^{2}\right]$. The straight line corresponds to an exponential decay with a characteristic decay time $\tau_{\mathrm{ch}}=4 \mathrm{~min}$. Note that after $20 \mathrm{~min}$ the correlations are at the noise level. (b) Log-log plot of the autocorrelation function of the absolute returns. The solid line is a power-law regression fit over the entire range, which gives an estimate of the power-law exponent, $\eta=0.29 \pm 0.05$. Better estimates of this exponent can be obtained from the power spectrum or from other more sophisticated methods.

$G$ now is correlated with $G$ at a time $\tau$ later, we measure that quantity as a function of $\tau$, and as the size of $\tau$ increases, the correlation decreases. It is remarkable that this decrease happens in a regular fashion. How do we interpret this decrease? If we put the autocorrelation function in logarithmic units and the time lag in linear units, we see that the data fall on an approximate straight line (Fig. 2a). This means that the function is decaying exponentially, which means it does indeed have a characteristic scale [13-15]. So the autocorrelation function is not scale invariant. This differs from 
systems near their critical points in which the autocorrelation functions are scale invariant.

\section{Statistical features of price fluctuations}

The decay time in this economic example is short (4 min), so one cannot easily "make money" on these correlations [13,14]. A little less well-known is the measure of the volatility $[14,15]$. One way to quantify volatility is to replace $G$ (the price change) with the absolute value of $G$. The data now are not at all linear on log-linear paper, but they are linear on $\log -\log$ paper (Fig. 2b). And, of course, a power-law $y=x^{p}$ is linear on $\log -\log$ paper, because $\log y=p \log x$. The slope of the $\log -\log$ plot $p$ is the value of the exponent. These exponents turn out to be fundamental quantities. In this case $p=-0.3$. The data are straight from about $200 \mathrm{~min}$ out to about $10^{5} \mathrm{~min}-\mathrm{a}$ range of almost 1000. With the data graphed we can see the approximate region in which the data are straight - the data are not straight over all regions. Qualitatively, we have known for a long time that there are long-range correlations in the volatility, e.g., volatility "clustering" and "persistence", but this graph helps quantify this known empirical fact.

So, if we cannot find an ordinary correlation near a critical point, we must try something else. For example, we might simply dump all of our data "on the floor". After we do that, the data no longer have time ordering nor do they have long- or short-range power-law correlations in the volatility of the autocorrelation function itself. Now we pick the data points up off the floor and make a histogram. Mandelbrot did this in 1963 with 1000 data points - a tiny number by today's standardsfor cotton-price fluctuations [12]. He demonstrated that those data were consistent with a Lévy distribution, i.e., a power-law distribution in that histogram-a so-called "fat tail".

In 1995, Mantegna and I decided to test this result using data with $\Delta t$ shorter than the daily data available in 1963 [13]. We used approximately 1 million data pointsthree orders of magnitude greater than Mandelbrot's data set. Instead of Mandelbrot's daily returns on cotton prices, we had returns approximately every $15 \mathrm{~s}$ on the S\&P 500 index. We found that on a log-linear plot (i) the histogram of the $G$ data points for the S\&P 500 clearly is not a Bachelier/Black-Scholes Gaussian, and (ii) although the center of the histogram agrees fairly well with Mandelbrot's Lévy distribution, it begins to disagree after a few standard deviations. This disagreement led us to develop a class of mathematical processes called truncated Lévy distributions - which has attracted the attention of a number of mathematicians, who have carried this concept far further than we could [16-21].

What about "universality", the notion in statistical physics that many laws seem to be remarkably independent of little details? A good example is that dramatically different materials behave exactly the same near their respective critical points. Binary mixtures, binary alloys, ferromagnets, even biological systems that involve switching, all behave 


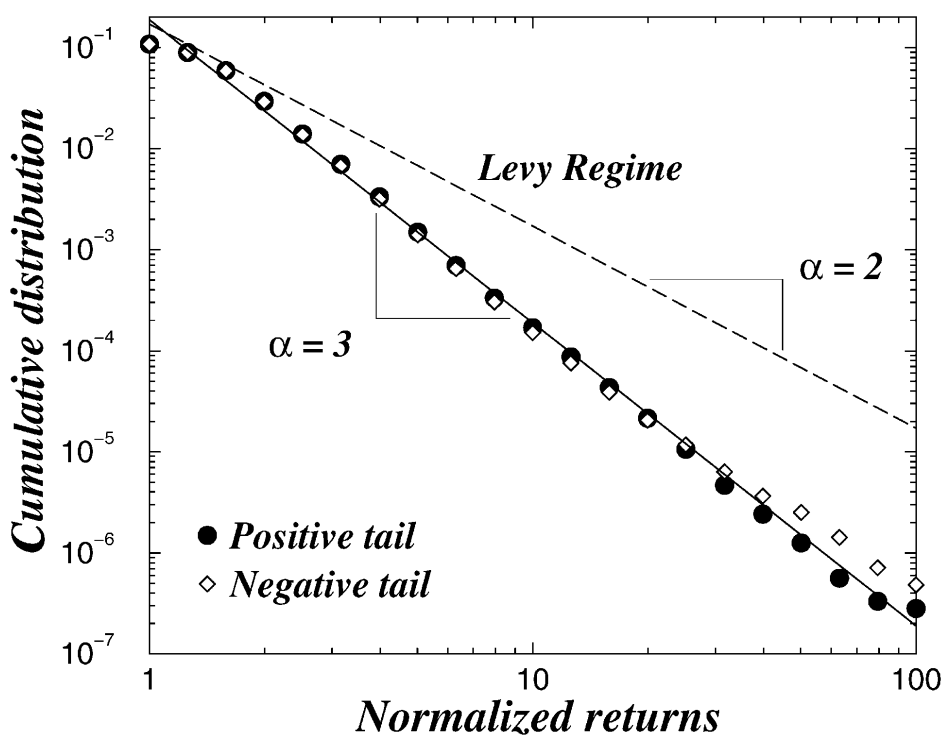

Fig. 3. Cumulative distributions of the positive and negative tails of the normalized returns of the 1000 largest companies in the TAQ database for the 2-year period 1994-1995. The solid line is a power-law regression fit in the region $2 \leqslant x \leqslant 80$.

the same way. We are finding the analog of this universality in economics. For example, Skjeltorp [22] did a study that utilized the Mantegna approach. Instead of 1,500,000 points from the S\&P 500 (15-s intervals spread over 6 years), Skjeltorp did a parallel study of the Norwegian stock exchange - and got almost exactly the same result.

We assumed that the reason we saw the truncated Lévy distribution while Mandelbrot did not was because we had more data - by three orders of magnitude. Gopikrishnan and Plerou recently acquired a data set three orders of magnitude larger still (of order $10^{9}$ ) - one that records every transaction of every stock. They found that when their data were graphed on log-log paper the result was linearity [23-26] (Fig. 3). This is the $\log$ of the cumulative distribution - the same quantity Mandelbrot plotted for cotton. But where Mandelbrot's straight line had a slope of about 1.7 (well inside the Lévy regime, which stops at slope 2.0), Gopikrishnan's straight line has a slope of $\approx 3.0$ (far outside the limit for a Lévy distribution). The fact that these data are approximately linear over two orders of magnitude means that fluctuations that are as much as 100 standard deviations are still conforming to the same law that describes the smaller fluctuations. This is reminiscent of the Gutenberg-Richter law that describes earthquakes. Thus it would seem that these very rare events, which are conventionally treated as totally unexpected and unexplainable, have a precise probability describable by the same law that describes much more common events. These rare events occur with a frequency 8 orders of magnitude less than the common, everyday event.

This means that Mandelbrot's results for cotton $\left(10^{3}\right.$ points $)$ are at total odds with Gopikrishnan's results for the S\&P 500 ( $10^{9}$ points $)$. Why this difference? Is it simply because Mandelbrot had too few data? Or do commodities intrinsically have fatter tails? 
In recent work with data from British Petroleum, it appears that commodity data may have a slightly smaller slope-consistent with the possibility that perhaps there is not one universal behavior for everything, but at least two separate universal behaviorsone for commodities and one for equities [27]. This smaller slope is still above 2, so the commodity data are not in the Lévy regime (even current data on cotton display a slope well above 2).

\section{Some similarities with diffusion in a tsunami wave}

Over this past year, we have been trying to understand these exponents using procedures similar to those used in critical phenomena, e.g., we relate one exponent to another and call the relation a scaling law, or we derive some microscopic model and call it renormalization group.

In particular, there appears to be an analog with anomalous diffusion. It is plausible that classical diffusion does not describe all random motion. The Brownian motion seen in the behavior of a grain of pollen in relatively calm water becomes something quite different if the grain of pollen is in a tsunami wave. The histograms would certainly be perturbed by a tsunami. A tsunami is an apt verbal metaphor for such economic "earthquakes" as the Merriwether disaster. So why not explore the stock market as an example of anomalous diffusion.

In one-dimensional classic diffusion, a particle moves at constant velocity until it collides with something. One calculates, e.g., the end position of the particle, and (of course) finds a Gaussian. Within a fixed time interval $\Delta t$, one might calculate a histogram for the number of collisions $p(N)$, and also find a Gaussian. And if one did a histogram of the variance $W^{2}$, one would also find a Gaussian. The fact that these are relatively narrow Gaussians means that there is a characteristic value, i.e., the width of that Gaussian, and that this is the basis for classical diffusion theory.

The corresponding quantity in the stock market to the displacement $x$ is the price. At each transaction there is a probability that the price will change, and after a given time horizon there is a total change $G$. We've seen the histogram of $G$ values - the cumulative obeyed an inverse cubic law, and therefore the pdf, by differentiation, obeys an inverse quartic law.

What about these histograms? Apparently no one had calculated these previously. Plerou et al. set about using the same data analyzed previously for $G$ to calculate the histograms of $N$ and $W^{2}$ (Fig. 4). They also found power laws-not Gaussians, as in classic diffusion. That means there is no characteristic scale for the anomalous diffusion case (there is a characteristic scale for the classic diffusion case), and for an obvious reason. If you are diffusing around in a medium-such as the "economic universe" we live in - in which the medium itself is changing, then the laws of diffusion change and, in particular, they adopt this scale-free form. Further, the exponents that describe $p(N)$ and $p\left(W^{2}\right)$ appear $[28,29]$ to be the analogs of exponents in critical phenomena in the sense that they seem to be related to one another in interesting ways. 

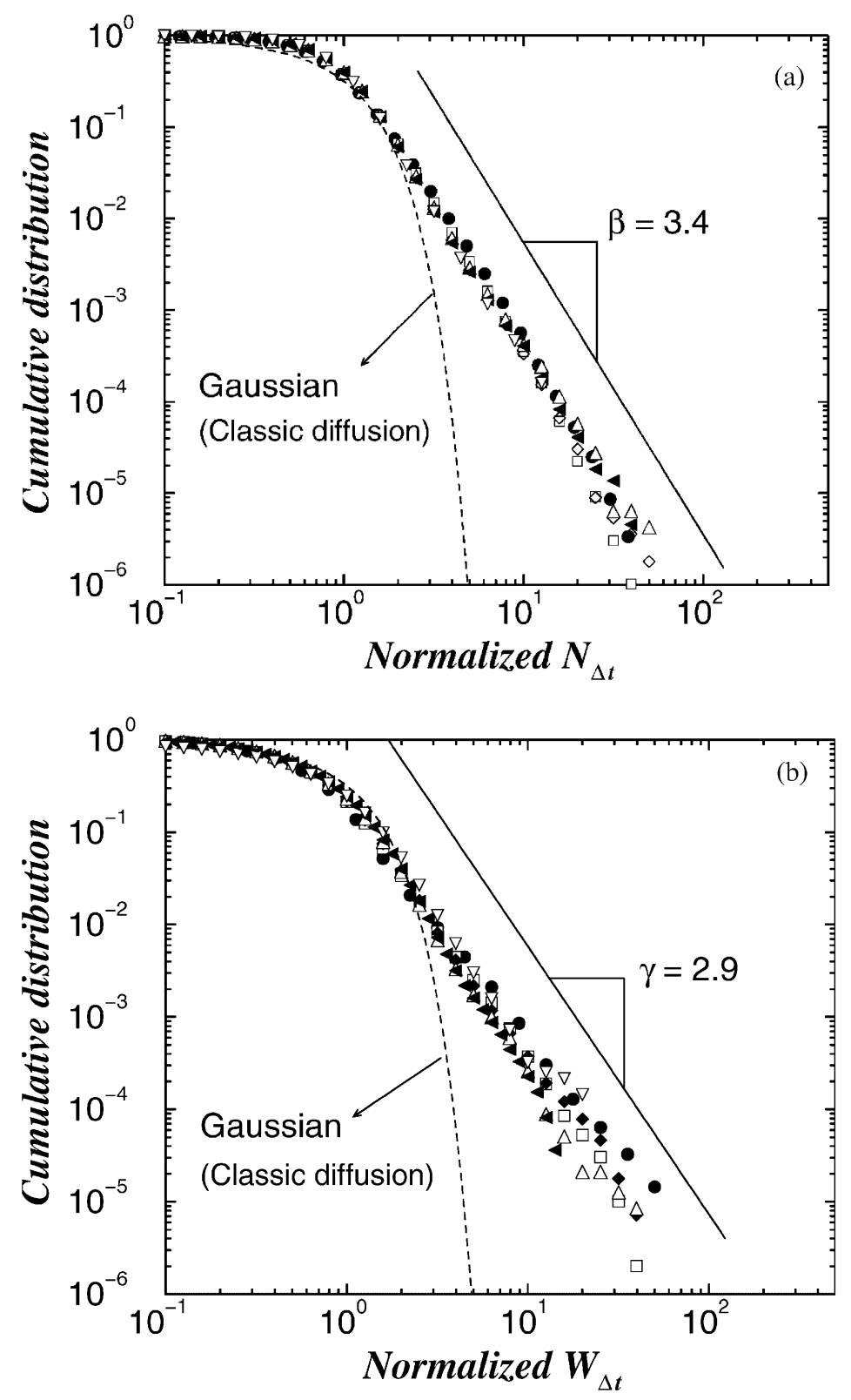

Fig. 4. (a) Log-log plot of the cumulative distribution of $N_{\Delta t}$ for the stocks in each of six groups defined by different levels of trading activity. Since each stock has a different average value of $\left\langle N_{\Delta t}\right\rangle$, we use a normalized number of transactions $n_{\Delta t} \equiv N_{\Delta t} /\left\langle N_{\Delta t}\right\rangle$. Each symbol shows the cumulative distribution $P\left\{n_{\Delta t}>x\right\}$ of the normalized number of transactions $n_{\Delta t}$ for all stocks in each group. An analysis of the exponents obtained by fits to the cumulative distributions $P\left\{N_{\Delta t}>x\right\}$ of each of the 1000 stocks yields an average value $\beta=3.40 \pm 0.05$. (b) $\log -\log$ plot of the cumulative distribution of $W_{\Delta t}$ for each of the six groups. (b) Since the average value $\left\langle W_{\Delta t}\right\rangle$ changes from one stock to another, we normalize $W_{\Delta t}$ by $\left\langle W_{\Delta t}\right\rangle$. Each symbol shows the cumulative distribution of the normalized $W_{\Delta t}$ for all stocks in each group. We analyze the power-law exponents $\gamma$ obtained by fits to the cumulative distributions of $W_{\Delta t}$ of each of the 1000 stocks separately and find an average value $\gamma=2.9 \pm 0.1$. 


\section{Some similarities with critical point phenomena}

Stock prices respond to fluctuations in demand, just as the magnetization of an interacting spin system responds to fluctuations in the magnetic field. Periods with large number of market participants buying the stock imply mainly positive changes in price, analogous to a magnetic field causing spins in a magnet to align. Recently, Plerou et al. [31] addressed the question of how stock prices respond to changes in demand. They quantified the relations between price change $G$ over a time interval $\Delta t$ and two different measures of demand fluctuations: (a) $\Phi$, defined as the difference between the number of buyer- and seller-initiated trades, and (b) $\Omega$, defined as the difference in number of shares traded in buyer- and seller-initiated trades. They find that the conditional expectations $\langle G\rangle_{\Phi}$ and $\langle G\rangle_{\Omega}$ of price change for a given $\Phi$ or $\Omega$ are both concave. They find that large price fluctuations occur when demand is very small - a fact which is reminiscent of large fluctuations that occur at critical points in spin systems, where the divergent nature of the response function leads to large fluctuations. Their findings are reminiscent of phase transitions in spin systems, where the divergent behavior of the response function at the critical point (zero magnetic field) leads to large fluctuations [11].

\section{Cross-correlations in price fluctuations of different stocks}

We know that a stock price does not vary in isolation from other stock prices, but that stock prices are correlated. That, of course, is not surprising because we know that (from the very beginning of this presentation) "in economics everything depends on everything else". How do we quantify these cross-correlations of one stock with another? If we take the $G$ values of four companies out of the 1000 that we have studied-corresponding to the shrinking or growing of each of these four companies in, say, a 30-min interval. How does the behavior of these four companies during that half-hour interval affect your response to their price activity? If two of the companies were Pepsi and Coke, there would probably be some correlation in their behaviors. One way to make money is to trade off these correlations. If you see one of them drop in price and the other does not, there is a good chance that the one that dropped will eventually come back up, will revert to the mean.

In order to quantify this, we begin by calculating a cross-correlation matrix. If we have 1000 firms, we have a $1000 \times 1000$ matrix. This large number of elements (1 million) does not frighten a physicist with a computer. Eugene Wigner applied random matrix theory 50 years ago to interpret the complex spectrum of energy levels in nuclear physics [30,32-42]. We do exactly the same thing, and apply random matrix theory. We find that certain eigenvalues of that $1000 \times 1000$ matrix deviate from the predictions of random matrix theory, which has not eigenvalues greater than an upper bound of $\approx 2.0$. Furthermore, the content of the eigenvectors corresponding to those eigenvalues correspond to well-defined business sectors. This is not surprising, but it 
does allow us to define business sectors without knowing anything about the separate stocks.

\section{Statistical physics and firm growth}

The economy is a little like a spin glass [43]. In the economy, each firm depends on every other firm, and the interactions are not short-ranged nor are they of uniform sign. For example, Ford Motor Company is in trouble because they have been selling their Explorer vehicle with extremely unsafe tires - and the price of their stock is going down. Prospective buyers purchase General Motors cars instead. There is a negative correlation between the stock prices of the two companies. But then General Motors needs to hire more workers to make a larger number of cars, and the McDonald's near the assembly plant has many more customers at lunchtime - a positive correlation in growth. Sometime later the situation may change again. So we can say that the "spins" all interact with one another, and that these interactions change as a function of time.

Nevertheless, the general idea of a critical phenomenon seems to work. If the firms were spread out in a kind of chain, the correlations among them would decay exponentially. Instead, the firms interact with each other much the same as critical phenomena interact with each other. This fact motivated a study carried out about 5 years ago by a group of physicists interacting with economist Michael Salinger [44-46]. They calculated the fluctuations in business firms from 1 year to the next. They found that if they broke the fluctuations into bins by size a tent-shaped distribution function was produced for each day of trading. The width of the tent was narrower for large firms than the width of the tent for small firms. This is not surprising, since a small firm has a potential to grow or shrink much more rapidly than a larger firm. When the widths of these tent-shaped distribution functions were plotted on log-log paper as a function of histogram size, the decreasing function turns out to be a straight line-corresponding to a power-law behavior in that function, and the exponent in that power-law is $\approx 0.2$. The linearity extends over a number of decades, indicating that the data collapse onto a single plot irrespective of scale. That was a new result, and this result appears to be quite robust - and has caught the attention of John Sutton [47], one of the leading economists at the London School of Economics, and number of other economists far more than anything we have done in finance.

\section{Universality in economic fluctuations}

Takayasu et al. have demonstrated that the above results are universal by moving outside the realm of US economies and studying firm behavior in other parts of the world [48].

Buldyrev et al. have shown that organizations (such as business firms) that are organized like trees will fluctuate in size [46]. The hierarchical structure is set up so that 
instructions from the top of the hierarchy propagate down to the branching lower levels of the structure. Within that structure is a disobedience factor-those lower down do not always obey the directives handed down from those above them. This factor is, of course, crucial to the survival of the system. If employees always did only and exactly what they were told, any small mistake put into the system by a manager would grow and do an increasing amount of damage as it propagated through the expanding tree structure of the organization. On the other hand, the probability of an instruction being disobeyed cannot be one - or chaos would result. So the propensity to disobey can be neither infinitesimal nor unity. The "obeying probability" needs to settle at a point at which the organization can maintain both its integrity and self-corrective flexibility. And the behavior of the exponent describing this probability is very similar to the behavior of critical exponents.

This result is fairly robust, not only as far as business firm fluctuations are concerned, but also in the size of countries. Lee et al. extend the same analysis used for business firms to countries - and with the same exponent [49]. Data can therefore be graphed on the same curve both for firms and for countries - where country size is measured by GDP.

We can see a similar pattern in the funding of university-based research. We researchers compete for research money the same way business firms compete for customers. Plerou et al. analyzed the funding of research groups over 17-year period in the same way fluctuations in firm size were analyzed [50]. The results were very similar with the data collapsing onto the same curve.

As a final example, we consider the case of fluctuating bird populations in North America. In this case the exponent is 0.35 instead of 0.2 . But, nevertheless, there seems to be some kind of property of contact organizations that we do not understand well [51].

\section{1. "Take-home message"}

So - what have we learned? Firstly, that the approach we have emphasized is an empirical approach where one first seeks to uncover features of the complex economy that are challenges to understand. We find that there are two new universal scaling models in economics: (i) the fluctuation of price changes of any stock market is characterized by a PDF which is a simple power law with exponent $\alpha+1=4$ that extends over $10^{2}$ standard deviations (a factor of $10^{8}$ on the $y$-axis); (ii) for a wide range of economic organizations, the histogram that shows how size of organization is inversely correlated to fluctuations in size with an exponent $\beta \approx 1 / 6$.

Neither of these two new laws has a firm theoretical foundation. This situation parallels the situation in the 1960s when the new field of critical phenomena also did not have a firm theoretical foundation for its new laws, but was awaiting the renormalization group. It is my hope that some of you in this room will rise to the challenge and try 
to find a firm theoretical foundation for the structure of the empirical laws that appear to be describing (i) finance fluctuations, and (ii) economic organizations.

\section{References}

[1] N.A. Criss, Review of Mantegna and Stanley, an introduction to econophysics, Physics Today 53 (2000) 12.

[2] R.N. Mantegna, H.E. Stanley, An Introduction to Econophysics: Correlations and Complexity in Finance, Cambridge University Press, Cambridge, 1999.

[3] J.P. Bouchaud, M. Potters, Theory of Financial Risk, Cambridge University Press, Cambridge, 2000.

[4] H. Levy, M. Levy, S. Solomon, Microscopic Simulation of Financial Markets, Academic Press, New York, 2000.

[5] B.M. Roehner, Hidden Collective Factors in Speculative Trading, Springer, Berlin, 2001.

[6] L. Bachelier, Théorie de la spéculation [Ph.D. thesis in mathematics], Ann. Sci. Ecole Norm. Sup. III-17 (1900) 21.

[7] S. Ghashgaie, W. Breymann, J. Peinke, P. Talkner, Y. Dodge, Turbulent cascades in foreign exchange markets, Nature 381 (1996) 767-770.

[8] R.N. Mantegna, H.E. Stanley, Turbulence and exchange markets, Nature 383 (1996) 587-588.

[9] R.N. Mantegna, H.E. Stanley, Stock market dynamics and turbulence: parallel analysis of fluctuation phenomena; Proceedings of the International Conference on Pattern Formation in Fluids and Materials, Physica A 239 (1997) 255-266.

[10] H.E. Stanley, Scaling, universality, and renormalization: three pillars of modern critical phenomena, Rev. Mod. Phys. 71 (1999) S358-S366.

[11] H.E. Stanley, Introduction to Phase Transitions and Critical Phenomena, Oxford University Press, Oxford, 1971.

[12] B.B. Mandelbrot, The variation of certain speculative prices, J. Business 36 (1963) 394-419.

[13] R.N. Mantegna, H.E. Stanley, Scaling behavior in the dynamics of an economic index, Nature 376 (1995) 46-49.

[14] Y. Liu, P. Gopikrishnan, P. Cizeau, M. Meyer, C.-K. Peng, H.E. Stanley, The statistical properties of the volatility of price fluctuations, Phys. Rev. E 60 (1999) 1390-1400.

[15] Z. Ding, C.W.J. Granger, R.F. Engle, A long memory property of stock market returns and a new model, J. Empirical Finance 1 (1993) 83-105.

[16] R.N. Mantegna, H.E. Stanley, Stochastic process with ultraslow convergence to a Gaussian: the truncated Lévy flight, Phys. Rev. Lett. 73 (1994) 2946-2949.

[17] B. Podobnik, P.Ch. Ivanov, Y. Lee, A. Chessa, H.E. Stanley, Truncated Lévy flights in the presence of variance correlations, Europhys. Lett. 50 (2000) 711-717.

[18] R.N. Mantegna, H.E. Stanley, Ultra-slow convergence to a Gaussian: the truncated Lévy flight, in: M.F. Shlesinger, G.M. Zaslavsky, U. Frisch (Eds.), Lévy Flights and Related Topics in Physics; Proceedings of the 1994 International Conference on Lévy Flights, Springer, Berlin, 1995, pp. 300-312.

[19] R.N. Mantegna, H.E. Stanley, Modeling of financial data: comparison of the truncated Lévy flight and the $\mathrm{ARCH}(1)$ and $\mathrm{GARCH}(1,1)$ processes; Proceedings of the International IUPAP Conference on Statistical Physics, Taipei, Physica A 254 (1998) 77-84.

[20] B. Podobnik, P.Ch. Ivanov, Y. Lee, H.E. Stanley, Scale-invariant truncated Lévy process, Europhys. Lett. 52 (2000) 491-497.

[21] P.Ch. Ivanov, B. Podobnik, Y. Lee, H.E. Stanley, Physica A 299 (2001) 154-160 [these proceedings].

[22] J.A. Skjeltorp, Scaling in the Norwegian stock market, Physica 283 (2001) 486-528.

[23] T. Lux, The stable paretian hypothesis and the frequency of large returns: an examination of major German stocks, Appl. Finan. Econ. 6 (1996) 463-475.

[24] P. Gopikrishnan, M. Meyer, L.A.N. Amaral, H.E. Stanley, Inverse cubic law for the distribution of stock price variations, Eur. Phys. J. B 3 (1998) 139-140.

[25] V. Plerou, P. Gopikrishnan, L.A.N. Amaral, M. Meyer, H.E. Stanley, Scaling of the distribution of price fluctuations of individual companies, Phys. Rev. E 60 (1999) 6519-6529.

[26] P. Gopikrishnan, V. Plerou, L.A.N. Amaral, M. Meyer, H.E. Stanley, Scaling of the distributions of fluctuations of financial market indices, Phys. Rev. E 60 (1999) 5305-5316. 
[27] K. Matia, P. Gopikrishnan, V. Plerou, L.A.N. Amaral, S. Goodwin, H.E. Stanley, Scaling for the distribution of the commodity market variations (preprint).

[28] V. Plerou, P. Gopikrishnan, L.A.N. Amaral, X. Gabaix, H.E. Stanley, Diffusion and economic fluctuations, Phys. Rev. E (Rapid Commun.) 62 (2000) 3023-3026.

[29] P. Gopikrishnan, V. Plerou, X. Gabaix, H.E. Stanley, Statistical properties of share volume traded in financial markets, Phys. Rev. E (Rapid Commun.) 62 (2000).

[30] M.L. Mehta, Random Matrices, Academic Press, Boston, 1991.

[31] V. Plerou, P. Gopikrishnan, X. Gabaix, H.E. Stanley, Quantifying stock price response to demand fluctuations, cond-mat 0106657.

[32] T. Guhr, A. Müller-Groeling, H.A. Weidenmüller, Random-matrix theories in quantum physics: common concepts, Phys. Rep. 299 (1998) 189-425.

[33] E.P. Wigner, On a class of analytic function from the quantum theory of collisions, Ann. Math. 53 (1951) 36-65.

[34] E.P. Wigner, On the statistical distribution of the widths and spacings of nuclear resonance levels, Proc. Cambridge Philos. Soc. 47 (1951) 790-798.

[35] E.P. Wigner, Results and theory of resonance absorption, in: Conference on Neutron Physics by Time-of-flight, Gatlinburg, Tennessee, 1956, pp. 59-70.

[36] M.L. Mehta, F.J. Dyson, Statistical theory of the energy levels of complex systems. V, J. Math. Phys. 4 (1963) 713-719.

[37] F.J. Dyson, The distribution of singular values of certain random matrices, Rev. Mexicana Física 20 (1971) 231.

[38] A.M. Sengupta, P.P. Mitra, Distributions of singular values for some random matrices, Phys. Rev. E 60 (1999) 3389-3392.

[39] L. Laloux, P. Cizeau, J.-P. Bouchaud, M. Potters, Noise dressing of financial correlation matrices, Phys. Rev. Lett. 83 (1999) 1469-1482.

[40] V. Plerou, P. Gopikrishnan, B. Rosenow, L.A.N. Amaral, H.E. Stanley, Universal and nonuniversal properties of financial cross-correlation matrices, Phys. Rev. Lett. 83 (1999) 1471-1475.

[41] P. Gopikrishnan, B. Rosenow, V. Plerou, H.E. Stanley, Quantifying and interpreting collective behavior in financial markets, Phys. Rev. E 64 (2001) 035106-1.

[42] V. Plerou, P. Gopikrishnan, B. Rosenow, L.A.N. Amaral, T. Guhr, H.E. Stanley, A random matrix approach to financial cross-correlations, Phys. Rev. E, submitted for publication.

[43] R.N. Mantegna, H.E. Stanley, Physics investigation of financial markets, in: F. Mallamace, H.E. Stanley (Eds.), Proceedings of the International School of Physics "Enrico Fermi", Course CXXXIV, IOS Press, Amsterdam, 1997.

[44] M.H.R. Stanley, L.A.N. Amaral, S.V. Buldyrev, S. Havlin, H. Leschhorn, P. Maass, M.A. Salinger, H.E. Stanley, Scaling behavior in the growth of companies, Nature 379 (1996) 804-806.

[45] L.A.N. Amaral, S.V. Buldyrev, S. Havlin, H. Leschhorn, P. Maass, M.A. Salinger, H.E. Stanley, M.H.R. Stanley, Scaling behavior in economics: I. Empirical results for company growth, J. Phys. I France 7 (1997) 621-633.

[46] S.V. Buldyrev, L.A.N. Amaral, S. Havlin, H. Leschhorn, P. Maass, M.A. Salinger, H.E. Stanley, M.H.R. Stanley, Scaling behavior in economics: II. Modeling of company growth, J. Phys. I France 7 (1997) 635-650.

[47] J. Sutton, The variance of firm growth rates: the 'scaling' puzzle, working paper, London School of Economics, 2000.

[48] H. Takayasu, K. Okuyama, Country dependence on company size distributions and a numerical model based on competition and cooperation, Fractals 6 (1998) 67-79.

[49] Y. Lee, L.A.N. Amaral, D. Canning, M. Meyer, H.E. Stanley, Universal features in the growth dynamics of complex organizations, Phys. Rev. Lett. 81 (1998) 3275-3278.

[50] V. Plerou, L.A.N. Amaral, P. Gopikrishnan, M. Meyer, H.E. Stanley, Similarities between the growth dynamics of university research and of competitive economic activities, Nature 400 (1999) $433-437$.

[51] T. Keitt, H.E. Stanley, Scaling in the dynamics of North American breeding-bird populations, Nature 393 (1998) 257. 